\title{
ROC curves show that the revelation effect is not a single phenomenon
}

\author{
MICHAEL F. VERDE and CAREN M. ROTELLO \\ University of Massachusetts, Amherst, Massachusetts
}

\begin{abstract}
The revelation effect describes the increased tendency to call items old when a recognition judgment is preceded by an incidental task. In theory, the effect could come about either from a more liberal response bias or from a change in underlying memory sensitivity. Using analyses of receiver-operating characteristic curves, we show that the revelation effect occurs for each of these reasons, but under different empirical conditions. A shift in response bias fully accounts for the revelation effect when revealed items are unrelated to the subsequent recognition probes. However, a change in memory sensitivity contributes to the effect when revealed items are identical to the recognition probes. Thus, the revelation effect encompasses at least two distinct phenomena.
\end{abstract}

Performing an incidental task just prior to a recognition judgment increases the tendency to claim that an item has been previously encountered. This memory illusion, the revelation effect, has been observed across a wide variety of conditions. Incidental tasks have included viewing the recognition probe in fragmentary or distorted form, typing it backward, or counting syllables and vowels (Luo, 1993; Watkins \& Peynircioğlu, 1990; Westerman \& Greene, 1998). The task need not even involve the recognition probe directly; the illusion is produced by anagrams of unrelated words and number strings, arithmetic problems, and memory span tests (Bornstein \& Neely, 2001; Niewiadomski \& Hockley, 2001; Verde \& Rotello, 2003; Westerman \& Greene, 1996, 1998). It has been assumed that the effects of these disparate task conditions represent a single phenomenon. In the present article, we report evidence from receiveroperating characteristic (ROC) curves that suggest that the revelation effect in fact encompasses at least two distinct phenomena.

A key question has been whether the revelation effect is due to response bias or a real change in the quality of retrieved information. Verde and Rotello (2003) have noted that signal detection theory offers a theoretical framework for examining this issue, as well as a means to distinguish between bias and memory change. The typical recognition or revelation procedure employs binary (old vs. new) judgments, providing a single pair of hit and false alarm rates per condition. By using the hit and false alarm rates, singlepoint sensitivity measures such as $d^{\prime}$ and $A^{\prime}$ are usually calculated. However, these measures make assumptions about the underlying distributions (Macmillan \& Creelman,

This research was supported by National Institutes of Health Research Grant MH60274-02 to C.M.R. and by National Institutes of Health Training Grant MH16745-19 to M.F.V. Correspondence concerning this article should be addressed to M. F. Verde, Department of Psychology, Box 37710, University of Massachusetts, Amherst, MA 01003-7710 (e-mail: mverde@psych.umass.edu).
1991, 1996), such as an equal-variance assumption, that are not typically justified (Macmillan \& Creelman, 1996; Ratcliff, Sheu, \& Gronlund, 1992). When the equal-variance assumption is violated, changes in response bias can lead to systematic changes in $d^{\prime}$ and $A^{\prime}$ even when no actual change in memory sensitivity has occurred. Thus, evaluation of the revelation effect with single-point measures alone is inadequate (see Verde \& Rotello, 2003, for more detail). However, more detailed information about the memory representation can be obtained from ROC curves.

Sample ROC curves are shown in Figure 1. They describe recognition for two conditions, revelation (prior to the memory judgment, subjects solved an anagram of an unrelated word) and baseline (no anagram), from Verde and Rotello (2003, Experiment 1). ${ }^{1}$ A useful aspect of ROC data is that they allow the separation of bias and sensitivity at a glance. Points that fall on the same curve represent different response biases but the same degree of sensitivity. On a given ROC curve, points closer to the lower left corner indicate more conservative responding (fewer old judgments). In contrast, ROC points that fall on different curves represent different degrees of sensitivity: Chance performance falls on the major diagonal, and increasing sensitivity moves the curve toward the upper left corner. It is clear in Figure 1 that the points for the revelation and the baseline conditions fall on a common curve; thus, revelation did not affect sensitivity in that experiment. Verde and Rotello argued that these findings suggest that the memory illusion is based on decision processes, rather than on changes in the quality of memory.

An important variation of the revelation manipulation not examined by Verde and Rotello (2003) is that in which the subject of the revelation task is the recognition probe itself. This variation gives the illusion its name - the recognition probe is revealed in some fashion that requires processing incidental to the memory judgment. However, following Westerman and Greene's (1996) surprising observation that processing an unrelated item also produces 


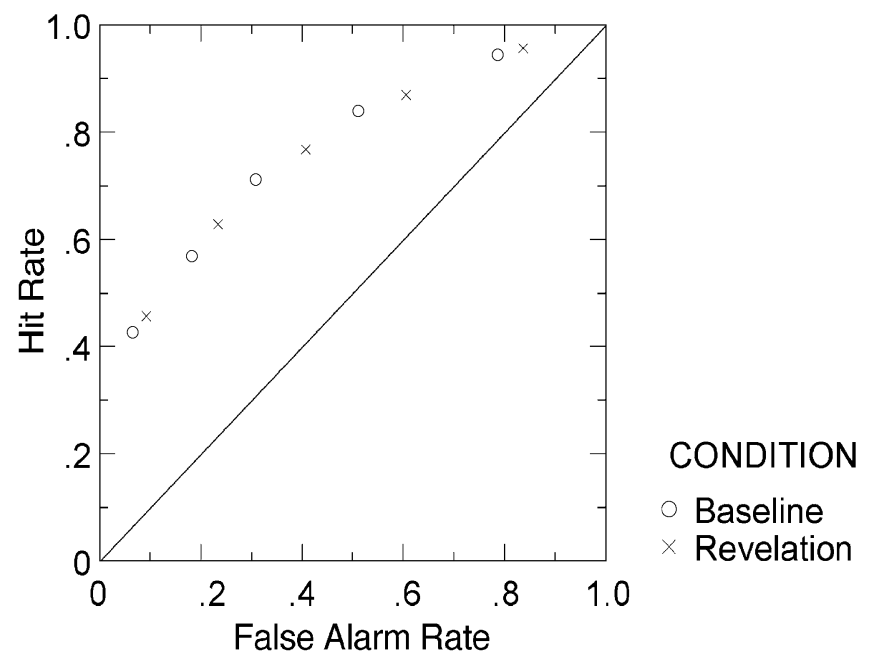

Figure 1. Sample receiver-operating characteristic curves (Experiment 1; Verde \& Rotello, 2003).

a robust revelation effect, it has become common to include only unrelated words in the revelation task. In the experiments to follow, we showed that these two task variations have qualitatively different effects on the subsequent memory judgment. In Experiment 1, we replicated our previous finding that solving an anagram of a word unrelated to the recognition probe affects response bias but not memory sensitivity. In subsequent experiments, we showed that solving an anagram of the recognition probe itself does affect sensitivity. This difference is critical to a theoretical interpretation of the revelation effect.

\section{EXPERIMENT 1}

Unscrambling a word or numerical string that is unrelated to a subsequent recognition probe has been shown to increase hits and false alarms without changing memory sensitivity (Verde \& Rotello, 2003). In Experiment 1, some recognition trials were preceded by an anagram created from a word that never appeared elsewhere in the study or test lists.

\section{Method}

Subjects. Twenty-two undergraduates from the University of Massachusetts at Amherst participated for course credit.

Materials and Design. The stimuli were drawn from a pool of 300 low-frequency eight-letter nouns ( $m=17 /$ million; Kučera \& Francis, 1967). The study list consisted of 50 words (40 critical words and 10 fillers placed at the beginning and end of the list). The test list consisted of 80 recognition probes, half from the study list and half new words. An additional 12 practice trials, created from filler items and new words, were placed at the beginning of the test. During the test, half of both the studied and the new items were preceded by an anagram created by scrambling the letters of a novel eight-letter word that was unrelated to the recognition probe (unrelated revelation condition). Every anagram could be unscrambled with the key 54687321 (the first letter of the anagram was the fifth letter in the unscrambled form, the second letter of the anagram was the fourth letter in the unscrambled form, and so on; for example, the anagram etmtpnoc would be solved to reveal contempt). The remain- ing test items served as the baseline: No anagrams preceded the recognition probe (no-revelation condition).

The assignment of words to condition and location within lists was randomized for each subject. List creation, stimulus presentation, and response collection were all computer controlled. The subjects were assigned to individual computers and testing rooms.

Procedure. The 30-min session consisted of a study phase followed by a test phase. At the beginning of the study phase, the subjects were told to learn the list of words for an upcoming memory test. The words were then presented individually on the computer screen, each for $2,000 \mathrm{msec}$, followed by a 500 -msec blank interval. During the test phase, each trial began with a fixation line displayed in the center of the screen for $500 \mathrm{msec}$. On half of the trials, a recognition probe then appeared in the center of the screen with the prompt "confidence? (1-6)" below it. The subjects were to decide whether the word had appeared in the study list. Judgments were made on a 6-point scale, ranging from 1 (very sure new) to 6 (very sure old). The recognition probe remained on the screen until a response was made. A 1,500-msec blank interval followed the response. On the other half of the trials, the recognition probe was preceded by an anagram probe. The subjects were to unscramble the anagram and type their answers. A 1,000-msec blank interval followed response completion, after which the recognition probe appeared. The subjects were given the anagram key and were instructed to use it to ensure $100 \%$ accuracy when solving the anagrams. Unrelated revelation and no-revelation condition trials were randomly intermixed within the test list.

\section{Results and Discussion}

For the anagrams, typing errors were defined as those in which no more than $25 \%$ of the letters were incorrect additions, omissions, or transpositions. More serious errors were rare $(<1 \%)$ because subjects were given the solution key. Anagram completion was not analyzed further.

Recognition performance was analyzed in two ways. First, for each condition, confidence ratings 1-3 were combined to form the new response category, and ratings 4-6 were combined to form the old response category. This yielded overall hit and false alarm rates that were submitted to an analysis of variance (ANOVA) in order to describe the 
general trends in the data. Second, the confidence ratings were used to construct an ROC curve for each experimental condition for each subject. The hits and false alarms for the confidence $=6$ category supplied the first (leftmost) point on the curve, the combined hits and false alarms for the 6 and 5 categories supplied the second point, and so on. A slope was then fit to the $z$-transformed ROC curve, using maximum likelihood estimation. This allowed calculation of the sensitivity measure $d_{a}$, which is appropriate when the variances of the distributions may be unequal (Macmillan \& Creelman, 1991):

$$
d_{a}=\left[2 /\left(1+\text { slope }^{2}\right)\right]^{1 / 2}[z(\mathrm{H})-\text { slope } \cdot z(\mathrm{FA})] .
$$

Recognition performance is summarized in Table 1 , and the full ROC curves are shown in Figure 2. An alpha level of .05 was used for all the statistical tests.

Solving an anagram just prior to recognition led to a revelation effect: There was an increase in both hits and false alarms. This conclusion was supported by a 2 (old/new probe) $\times 2$ (condition) repeated measures ANOVA that yielded significant main effects for both probe type $\left[F(1,21)=139.32, M S_{\mathrm{e}}=0.027, p<.001\right]$ and condition $\left[F(1,21)=17.46, M S_{\mathrm{e}}=0.016, p<.001\right]$. The interaction of probe type and condition was not significant. There was no significant difference in recognition sensitivity between the unrelated revelation $\left(d_{a}=1.13\right)$ and the no-revelation $\left(d_{a}=1.14\right)$ conditions. These results replicate Verde and Rotello (2003) in showing that changes in response bias can fully describe the revelation effect that occurs when words unrelated to the recognition probe are revealed. Memory sensitivity was unaffected by revelation in this experiment.

\section{EXPERIMENT 2}

Experiment 2 was identical in all respects to Experiment 1 , save for the identity of the anagram in the revelation condition. Here, the recognition probe itself was scrambled and served as the anagram on that trial.
Table 1

\begin{tabular}{|c|c|c|c|c|c|}
\hline Experiment & Condition & $\mathrm{H}$ & FA & $d_{a}$ & $S E$ \\
\hline \multirow[t]{2}{*}{1} & No revelation & .72 & .28 & 1.14 & 0.11 \\
\hline & Unrelated revelation & .80 & .42 & 1.13 & 0.11 \\
\hline \multirow[t]{2}{*}{2} & No revelation & .78 & .32 & 1.25 & 0.11 \\
\hline & Identical revelation & .80 & .51 & 0.88 & 0.12 \\
\hline \multirow[t]{2}{*}{3} & Unrelated revelation & .76 & .34 & 1.25 & 0.09 \\
\hline & Identical revelation & .77 & .42 & 1.04 & 0.11 \\
\hline \multirow[t]{3}{*}{4} & No revelation & .74 & .29 & 1.13 & 0.08 \\
\hline & Unrelated revelation & .80 & .39 & 1.20 & 0.09 \\
\hline & Identical revelation & .76 & .45 & 0.97 & 0.08 \\
\hline
\end{tabular}

Note-Unrelated revelation, anagram created from a novel word unrelated to the recognition probe; identical revelation, anagram created from the recognition probe.

\section{Method}

Subjects. Twenty-two undergraduates from the University of Massachusetts at Amherst participated for course credit.

Materials and Design. The materials and design were identical to those in Experiment 1. However, anagrams were created by scrambling the recognition probes. Test trials in which the recognition probe was preceded by an anagram were designated the identical revelation condition.

Procedure. The procedure was identical to that in Experiment 1.

\section{Results and Discussion}

There were very few serious errors $(<1 \%)$ in the completion of anagrams. As before, recognition performance was analyzed first in terms of overall hit and false alarm rates and then by examining the ROC curves constructed from recognition confidence ratings. Recognition performance is summarized in Table 1, and the full ROC curves are shown in Figure 3.

Solving an anagram just prior to recognition led to a revelation effect: There was a small increase in hits and a much larger increase in false alarms. This conclusion was supported by a 2 (old/new probe) $\times 2$ (condition) repeated measures ANOVA that yielded significant main effects for both probe type $\left[F(1,21)=164.23, M S_{\mathrm{e}}=0.019, p<\right.$

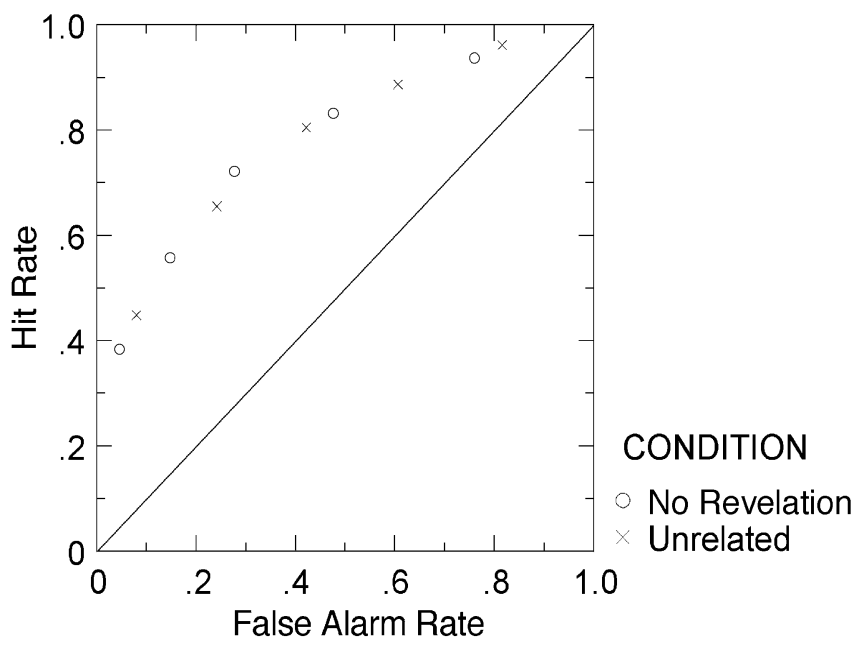

Figure 2. Experiment 1: Receiver-operating characteristic data. 


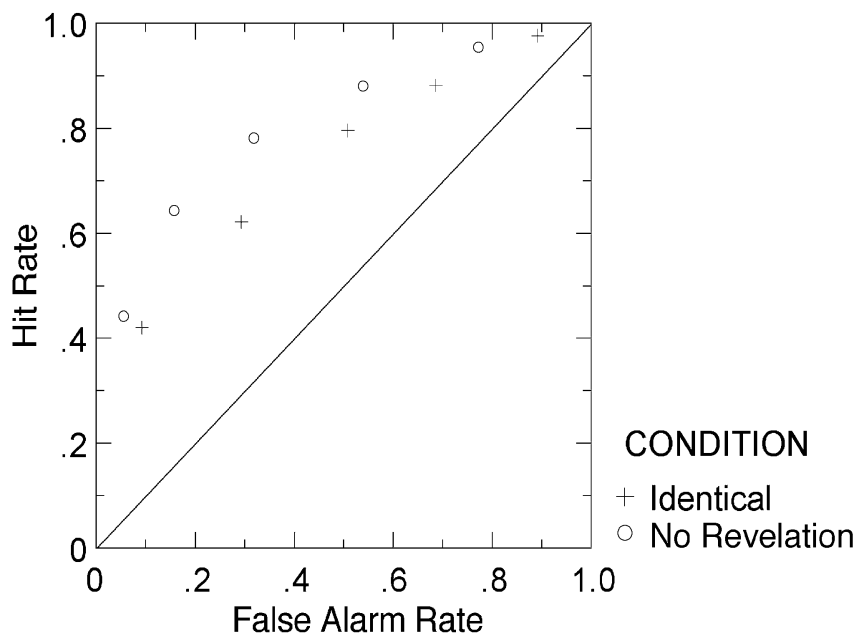

Figure 3. Experiment 2: Receiver-operating characteristic data.

$.001]$ and condition $\left[F(1,21)=14.58, M S_{\mathrm{e}}=0.016, p<\right.$ $.01]$, as well as an interaction between probe type and condition $\left[F(1,21)=16.15, M S_{\mathrm{e}}=0.010, p<.01\right]$. In contrast to the results of Experiment 1, in this experiment memory sensitivity was reduced in the identical revelation condition $\left(d_{a}=0.88\right)$, as compared with the no-revelation condition $\left[d_{a}=1.25 ; t(21)=3.34, p<.01\right]$. The difference in memory sensitivity can be seen in Figure 3 : The ROC points from the two conditions clearly fall on different curves. Thus, not only did solving an anagram increase the likelihood of responding old, it also had an adverse effect on accuracy.

\section{EXPERIMENT 3}

In order to directly compare the unrelated and identical revelation conditions, as well as to ensure that their differences were not the result of subjects having adopted dif- ferent judgment strategies between experiments, both conditions were included in Experiment 3, which was identical in all other respects to the previous two experiments.

\section{Method}

Subjects. Thirty-two undergraduates from the University of Massachusetts at Amherst participated for course credit.

Materials and Design. The materials and design were identical to those in Experiment 1. However, every recognition probe was preceded by an anagram. For half of the trials, the anagram was created from a novel word unrelated to the recognition probe (unrelated revelation condition). For the other half, the anagram was created by scrambling the recognition probe (identical revelation condition).

Procedure. The procedure was identical to that in Experiment 1.

\section{Results and Discussion}

There were very few serious errors $(<1 \%)$ in the completion of anagrams. Recognition performance is summarized in Table 1, and the full ROC curves are shown in Figure 4.

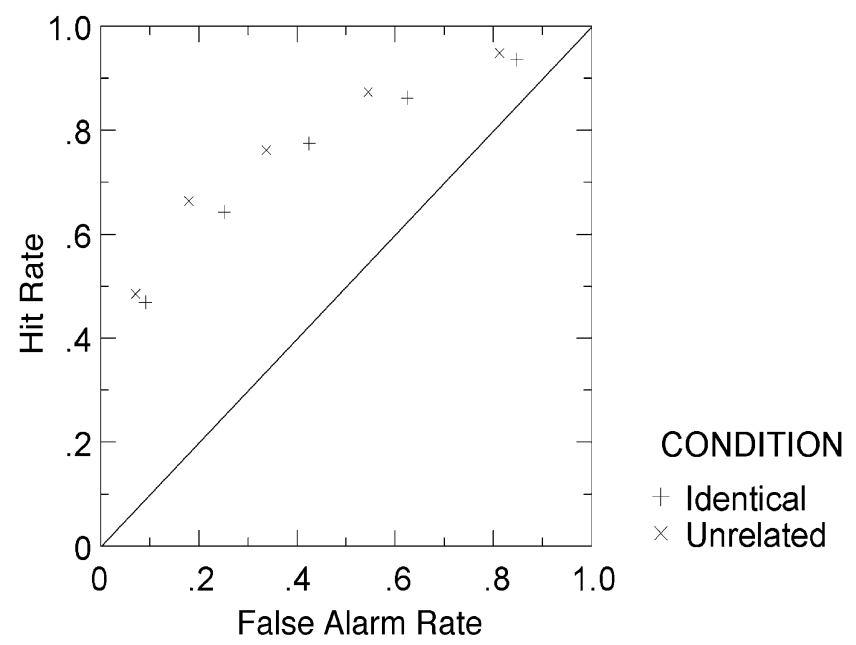

Figure 4. Experiment 3: Receiver-operating characteristic data. 
Unrelated and identical anagrams had different effects on the recognition judgment: Identical anagrams resulted in slightly more hits and many more false alarms. This conclusion was supported by a 2 (old/new probe) $\times 2$ (revelation condition) repeated measures ANOVA that yielded a significant main effect for probe type $[F(1,31)=139.54$, $\left.M S_{\mathrm{e}}=0.034, p<.001\right]$, a marginally significant effect of condition $\left[F(1,31)=3.57, M S_{\mathrm{e}}=0.023, p<.10\right]$, and a significant interaction between probe type and condition $\left[F(1,31)=4.54, M S_{\mathrm{e}}=0.010, p<.05\right]$. In accord with the results of Experiment 2, there was a significant reduction in memory sensitivity in the identical revelation condition $\left(d_{a}=1.04\right)$, as compared with the unrelated revelation condition $\left[d_{a}=1.25 ; t(31)=2.41, p<.05\right]$. These results support the conclusions that although a shift in response bias fully accounts for revelation effects based on revealed items that are unrelated to the recognition probes, it is insufficient to account for revelation effects based on revealed items that are identical to the recognition probes. In the latter case, a change in memory sensitivity also contributes to the revelation effect.

\section{EXPERIMENT 4}

The revelation effect is attenuated or absent when the recognition judgment involves retrieval of specific episodic information, such as with associative recognition or plurality judgments (Cameron \& Hockley, 2000; Westerman, 2000). Thus, the effect seems to be localized to judgments of nonspecific familiarity. Isolating the illusion to this memory process is important for theoretical interpretation, especially in the context of dual-process theories of recognition (for a review, see Yonelinas, 2002). The goal of Experiment 4 was to confirm that both identical and unrelated revelation tasks affect only familiarity. In addition, it served as a within-subjects replication of Experiments $1-3$.

The subjects studied two distinct lists, labeled "List 1" and "List 2." During the test, they first decided whether a recognition probe had appeared on either list. If a word was judged old, they also indicated the list in which the word had appeared. List discrimination judgments usually include a recollective component (Rotello \& Heit, 2000); thus, if revelation affects recollection, there should be differences in the subjects' ability to make source judgments as a function of revelation condition. The test included unrelated revelation, identical revelation, and no-revelation conditions.

\section{Method}

Subjects. Twenty-nine undergraduates from the University of Massachusetts at Amherst participated for course credit.

Materials and Design. The materials and design were identical to those in Experiment 1, except as indicated. The study items consisted of two separate lists (List 1 and List 2) of 30 words each. An additional filler word was placed at the beginning and end of each list. The test list consisted of 96 recognition probes, 48 studied words, and 48 new words. An additional eight practice trials, created from filler and new words, were placed at the beginning of the test. An equal number of studied and new words and an equal number of List 1 and List 2 words were assigned to the three test conditions: unrelated revelation, identical revelation, and no revelation.

Procedure. The 30-min session consisted of a study phase followed by a test phase. At the beginning of the study phase, the subjects were told to learn the two lists of words for an upcoming memory test and were warned that they would be asked about list membership. They were then shown List 1 , followed by List 2 . The words were presented individually on the computer screen, each for $2,000 \mathrm{msec}$, followed by a 500 -msec blank interval. The test procedure was similar to that in Experiment 1. However, on trials in which the subjects indicated that the probe was old (confidence rating 4-6), they were further asked to indicate list membership using the " 1 " and "2" keys for List 1 and List 2, respectively. Unrelated revelation, identical revelation, and no-revelation condition trials were randomly intermixed within the test list.

\section{Results and Discussion}

There were few serious errors $(<1 \%)$ in the completion of anagrams. Recognition performance is summarized in Table 1, with the full ROC curves shown in Figure 5.

The revelation task had no effect on source judgments. There were no significant differences in list discrimina-

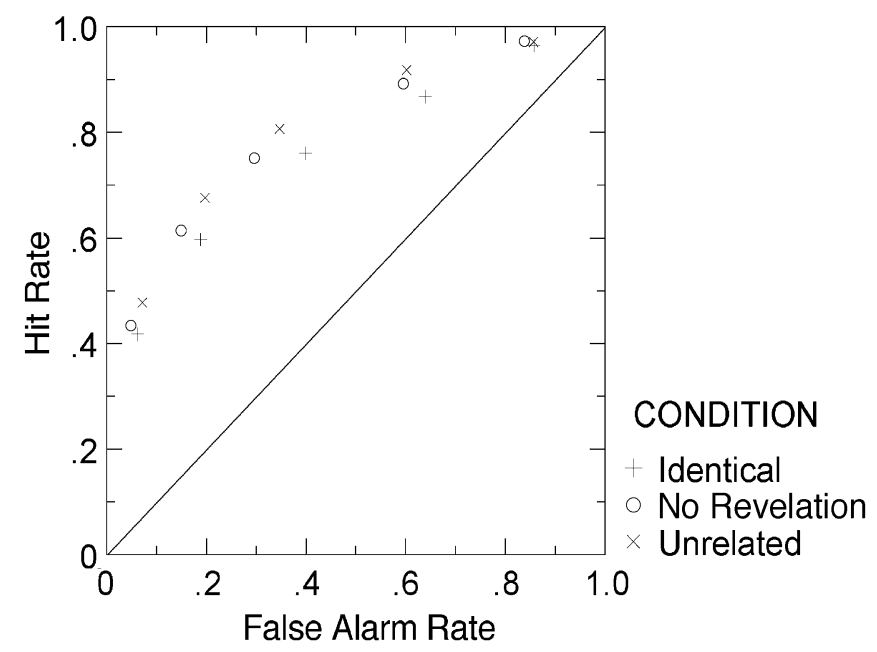

Figure 5. Experiment 4: Receiver-operating characteristic data. 
tion accuracy between the unrelated revelation (.59), identical revelation (.60), and no-revelation (.58) conditions $\left[F(2,56)=0.18, M S_{\mathrm{e}}=0.018\right]$. This is consistent with other findings that suggest that the revelation effect is associated primarily with judgments of nonspecific familiarity (Cameron \& Hockley, 2000; LeCompte, 1995; Westerman, 2000). Despite the null effect on source judgments, old-new confidence ratings replicated the patterns observed in the previous experiments.

Although both unrelated and identical anagrams produced a revelation effect, the changes in hits and false alarms were not identical. A 2 (old/new probe) $\times 3$ (condition) repeated measures ANOVA yielded significant main effects for both probe type $\left[F(1,28)=319.32, M S_{\mathrm{e}}=\right.$ $0.021, p<.001]$ and condition $\left[F(2,56)=6.39, M S_{\mathrm{e}}=\right.$ $0.022, p<.01]$, as well as a significant interaction between probe type and condition $\left[F(1,31)=6.47, M S_{\mathrm{e}}=0.011\right.$, $p<.01]$.

Pairwise comparisons between conditions revealed that recognition sensitivity was significantly lower after identical revelation $\left(d_{a}=0.97\right)$ than after either unrelated revelation $\left(d_{a}=1.21\right)$ or no revelation $\left(d_{a}=1.13\right)$ and that the latter two conditions did not differ reliably [identical revelation vs. no revelation, $t(28)=2.29, p<.05$; identical revelation vs. unrelated revelation, $t(28)=2.20, p<$ .05 ; unrelated revelation vs. no revelation, $t(28)=0.70]$. These results replicate within a single experiment the reduction in memory sensitivity due to identical revealed items and the bias shift (and lack of sensitivity change) due to unrelated revealed items.

\section{GENERAL DISCUSSION}

Memory illusions can reveal properties of the memory system not otherwise apparent under ordinary circumstances. With the revelation effect, the question of general interest is whether incidental tasks that appear close in time to a memory judgment actually change the quality of retrieved information (memory sensitivity) or affect only how people use that information (response bias). The present results suggest that there may not be a single correct answer, because, contrary to previous assumptions, the revelation effect is not a single phenomenon. With revelation items that are unrelated to the recognition probe, there is no effect of revelation on recognition sensitivity. A change in response bias can fully account for the increase in old response rates. This is not true when the revelation item is identical to the recognition probe.

It should be noted that under restricted conditions, a change in familiarity can be indistinguishable from a simple bias shift in ROC data. Specifically, if the revelation task causes both old and new items to increase in memory strength, so that the distance between the means of the strength distributions and the ratio of their standard deviations remains constant, there will be no change in sensitivity. The present data alone do not rule out this possibility in the unrelated revelation condition. However, data from other studies in which unrelated revelation items were used argue against the familiarity change interpretation. First, the size of the revelation effect can be manipulated by factors not obviously related to memory strength. The revelation effect produced by rare words and nonwords disappears when they are mixed with common words at study and test (Hockley \& Niewiadomski, 2001), and the revelation effect produced by nonwords may be greater or smaller than that of words, depending on when the revelation item is presented relative to the recognition probe (Whittlesea \& Williams, 2001). On the other hand, factors that should affect memory strength, such as prior exposure or the similarity of revealed items to the contents of memory, have no apparent effect on the magnitude of the revelation effect (Niewiadomski \& Hockley, 2001; Verde $\&$ Rotello, 2003). These prior results argue in favor of a bias change interpretation of the unrelated item revelation effect.

Why an unrelated revelation task should produce a liberal response bias remains unclear. A possibility offered by Niewiadomski and Hockley (2001) is that the revelation task causes one to temporarily lose track of the appropriate criterion setting. Another, offered by Hicks and Marsh (1998), is based on Hirshman's (1995) observation that response bias becomes more liberal as the difficulty of the recognition test increases. If subjects hold the (mistaken) belief that the revelation task interferes with the memory task, they may set their decision criterion accordingly.

With revelation items that are identical to the recognition probe, the revelation effect represents a real decline in memory accuracy and, as such, cannot be accounted for by response bias alone. Although the source of this decline remains to be explored, one possibility is that incidental processing of the recognition probe increases its familiarity. If this also increases the variability of the familiarity distributions, the resulting decline in the signalto-noise ratio would lead to a drop in accuracy.

\section{REFERENCES}

Bornstein, B. H., \& NeEly, C. B. (2001). The revelation effect in frequency judgment. Memory \& Cognition, 29, 209-213.

Cameron, T. E., \& Hockley, W. E. (2000). The revelation effect for item and associative recognition: Familiarity versus recollection. Memory \& Cognition, 28, 176-183.

HiCKS, J. L., \& MARSH, R. L. (1998). A decrement-to-familiarity interpretation of the revelation effect from forced-choice tests of recognition memory. Journal of Experimental Psychology: Learning, Memory, \& Cognition, 24, 1105-1120.

HiRshman, E. (1995). Decision processes in recognition memory: Criterion shifts and the list-strength paradigm. Journal of Experimental Psychology: Learning, Memory, \& Cognition, 21, 302-313.

HoCKLEY, W. E., \& NIEWIADOMSKI, M. W. (2001). Interrupting recognition memory: Tests of a criterion-change account of the revelation effect. Memory \& Cognition, 29, 1176-1184.

KučERA, H., \& FrANCIS, W. N. (1967). Computational analysis of presentday American English. Providence, RI: Brown University Press.

LeCompte, D. C. (1995). Recollective experience in the revelation effect: Separating the contributions of recollection and familiarity. Memory \& Cognition, 23, 324-334.

Luo, C. R. (1993). Enhanced feeling of recognition: Effects of identifying and manipulating test items on recognition memory. Journal of Experimental Psychology: Learning, Memory, \& Cognition, 13, 405-413. 
Macmillan, N. A., \& Creelman, C. D. (1991). Detection theory: A user's guide. New York: Cambridge University Press.

Macmillan, N. A., \& Creelman, C. D. (1996). Triangles in ROC space: History and theory of "nonparametric" measures of sensitivity and response bias. Psychonomic Bulletin \& Review, 3, 164-170.

Niewiadomski, M. W., \& HockLey, W. E. (2001). Interrupting recognition memory: Tests of familiarity-based accounts of the revelation effect. Memory \& Cognition, 29, 1130-1138.

Ratcliff, R., Sheu, C. F., \& GRONLund, S. D. (1992). Testing global memory models using ROC curves. Psychological Review, 99, 518-535.

Rotello, C. M., \& Heit, E. (2000). Associative recognition: A case of recall-to-reject processing. Memory \& Cognition, 28, 907-922.

Verde, M. F., \& Rotello, C. M. (2003). Does familiarity change in the revelation effect? Journal of Experimental Psychology: Learning, Memory, \& Cognition, 29, 739-746.

WATKIns, M. J., \& PEYNiRCIOǦLU, Z. F. (1990). The revelation effect: When disguising test items induces recognition. Journal of Experimental Psychology: Learning, Memory, \& Cognition, 16, 1012-1020.

WESTERMAN, D. L. (2000). Recollection-based recognition eliminates the revelation effect in memory. Memory \& Cognition, 28, 167-175.

Westerman, D. L., \& Greene, R. L. (1996). On the generality of the revelation effect. Journal of Experimental Psychology: Learning, Memory, \& Cognition, 22, 1147-1153.

WESTERMAN, D. L., \& GREENE, R. L. (1998). The revelation that the revelation effect is not due to revelation. Journal of Experimental Psychology: Learning, Memory, \& Cognition, 24, 377-386.

Whittlesea, B. W. A., \& Williams, L. D. (2001). The discrepancyattribution hypothesis: I. The heuristic basis of feelings and familiarity. Journal of Experimental Psychology: Learning, Memory, \& Cognition, 27, 3-13.

YoNELINAS, A. P. (2002). The nature of recollection and familiarity: A review of 30 years of research. Journal of Memory \& Language, $\mathbf{4 6}$, 441-517.

\section{NOTE}

1. Verde and Rotello (2003) reported only the summary statistics $d_{a}$ and the slope of the $z$ ROC.

(Manuscript received March 26, 2003; revision accepted for publication June 30, 2003.) 